\title{
Synthesis and characterisation of hierarchically structured titanium silicalite-1 zeolites with large intracrystalline macropores
}

\author{
Tobias Weissenberger, " [a] Rainer Leonhardt, ${ }^{[a]}$ Benjamin Apeleo Zubiri, [b] Martina Pitínová-Štekrová, \\ [c] Thomas L. Sheppard, ${ }^{[\mathrm{d}, \mathrm{i}]}$ Bastian Reiprich, ${ }^{[a]}$ Jürgen Bauer, ${ }^{[e]}$ Ralf Dotzel, [e] Maik Kahnt, [g, h] \\ Andreas Schropp, ${ }^{[g]}$ Christian G. Schroer, ${ }^{[g, h]}$ Jan-Dierk Grunwaldt, ${ }^{[d, ~ i] ~ J o h n ~ L . ~ C a s c i, ~}{ }^{[f]}$ Jiř́ Čejka, ${ }^{[j]}$ \\ Erdmann Spiecker, ${ }^{[b]}$ and Wilhelm Schwieger ${ }^{[a]}$
}

\begin{abstract}
We report the successful synthesis of hierarchically structured titanium silicalite-1 (TS-1) with large intracrystalline macropores by steam-assisted crystallisation of mesoporous silica particles. The macropore topology was imaged in 3D using electron tomography and synchrotron radiation-based ptychographic Xray computed tomography, revealing interconnected macropores within the crystals accounting for about $30 \%$ of the particle volume. The study of the macropore formation mechanism revealed that the mesoporous silica particles act as a sacrificial macropore template during the synthesis. Silicon to titanium ratio of the macroporous TS-1 samples was successfully tuned from 100 to 44 . The hierarchically structured
\end{abstract}

[a] T. Weissenberger, R. Leonhardt, B. Reiprich, W. Schwieger Institute of Chemical Reaction Engineering

University of Erlangen-Nuremberg

Egerlandstr. 3, 91058 Erlangen, Germany

E-mail: tobias.weissenberger@fau.de

[b] B. Apeleo Zubiri, E. Spiecker

Institute of Micro- and Nanostructure Research (IMN) \& Center for

Nanoanalysis and Electron Microscopy (CENEM)

University of Erlangen-Nuremberg

Cauerstr. 6, 91058 Erlangen, Germany

[c] M. Pitínová-Štekrová

J. Heyrovsky Institute of Physical Chemistry

Czech Academy of Sciences

Dolejškova 2155/3, 182 23, Prague 8, Czech Republic.

[d] T. L. Sheppard, J.-D. Grunwaldt

Institute of Catalysis Research and Technology

Karlsruhe Institute of Technology

Hermann-von-Helmholtz Platz 1, 76344 Eggenstein-Leopoldshafen

Germany

[e] J. Bauer, R. Dotzel

Johnson Matthey Catalysts (Germany) GmbH

Bahnhofstr. 43, 96257 Redwitz, Germany

[f] J. L. Casci

Johnson Matthey Technology Centre

PO Box 1, Belasis Avenue, Billingham, TS23 1LB, United Kingdom

[g] M. Kahnt, A. Schropp, C. G. Schroer

Deutsches Elektronen-Synchrotron DESY

Notkestraße 85, 22607 Hamburg, Germany

[h] C. G. Schroer

Department Physik

Universität Hamburg

Luruper Chaussee 149, 22761 Hamburg, Germany

[i] T. L. Sheppard, J.-D. Grunwaldt

Institute for Chemical Technology and Polymer Chemistry

Karlsruhe Institute of Technology

Engesserstr. 20, 76131 Karlsruhe, Germany

[i] J. Čejka

Department of Physical and Macromolecular Chemistry

Charles University in Prague

Hlavova 8, 12843 Prague 2, Czech Republic

Supporting information for this article is given via a link at the end of the document.((Please delete this text if not appropriate))
TS-1 exhibited high activity in the liquid phase epoxidation of 2octene with hydrogen peroxide. The hierarchically structured TS1 surpassed a conventional nano-sized TS-1 sample in terms of alkene conversion and showed comparable selectivity to the epoxide. The flexible synthesis route described here can be used to prepare hierarchical zeolites with improved mass transport properties for other selective oxidation reactions.

\section{Introduction}

Titanium zeolites have been subject to research since the discovery of titanium silicalite-1 (TS-1) by Taramasso and coworkers in 1983. [1] The isomorphic substitution of silicon for titanium results in the formation of isolated, tetrahedrally coordinated titanium sites in the zeolite framework. In contrast to aluminium, titanium shows the same formal valence state as silicon, resulting in an equal charge replacement of silicon by titanium in the resulting zeolite.

The isolated titanium acts as an active site for the oxidation of various organic molecules with hydrogen peroxide under mild reaction conditions.$^{[2]}$ Due to its activity, selectivity and stability TS-1 finds industrial application as a catalyst for the epoxidation of propene to propene oxide, the ammoxidation of cyclohexanone to cyclohexanone oxime and the hydroxylation of phenol to hydroquinone and catechol. [3]

However, the small micropores of zeolites (typically $<1 \mathrm{~nm}$ ) limit possible applications. The conversion of bulky molecules too large to enter the micropores is only possible on the relatively small number of active sites located on the external surface of the crystals, leading to very low activity. Furthermore, the intracrystalline diffusion of reactants to the active sites confined in the micropores takes place at a very low rate resulting in limitation of the observable reaction rate by the slow mass transport.

In order to overcome such mass transport limitations in zeolites, different approaches have been proposed in the literature focusing on improving accessibility for bulky substrates to the active sites. To achieve this, either the pore size of the zeolite can be increased therefore enhancing the diffusion rate of substrates into the zeolite, ${ }^{[4]}$ or the diffusion time can be reduced by a reduction of the characteristic diffusion path length..$^{[5]}$

The reduction of the characteristic diffusion path length is possible by reduction of the crystal dimensions in three or two dimensions, resulting in nanosized or layered like zeolites, respectively. [6] Nano-sized zeolite crystals offer relatively short diffusion path lengths in the micropores, effectively enhancing the mass transport. Furthermore, the larger external surface area increases the reaction rate for the conversion of bulky molecules. ${ }^{[7]} \mathrm{Nano}$ 
materials, however, come with some problems regarding their separation, handling, stability and their possible negative effects on human health. Larger crystals can also offer a reduced diffusion path length for example by introducing an additional network (e.g. mesopores) of larger pores interconnected to the micropores. ${ }^{[8]}$ The resulting hierarchically structured zeolites combine the stability and shape selectivity of the micropores with the improved mass transport properties of meso- or macropores. Hierarchically structured TS-1 zeolites have already demonstrated superior catalytic performance in different reactions, such as hydroxylation of phenol, ${ }^{[9]}$ epoxidation of 1-octene, ${ }^{[10]}$ epoxidation of cyclohexene ${ }^{[11]}$ and oxidation of thiophene. ${ }^{[12]}$

The preparation of hierarchically structured zeolites has been subject to research for many years and can be divided into topdown and bottom-up processes.

Most of the bottom-up processes for the preparation of hierarchically structured TS-1 rely on the templating effect of a hard or soft template to generate additional intra- or intercrystalline porosity. Carbon materials are commonly used as hard templates to generate additional mesopores. ${ }^{[9,13]}$ Soft templating typically utilises surfactants or organosilanes during the synthesis of the titanium-containing zeolite to generate mesopores. ${ }^{[12,14]}$ Nano-sized TS-1 crystals have also been used for the preparation of hierarchical materials by self-assembly. ${ }^{[15]}$ Another synthesis approach is the use of silanised proto-zeolitic units for the synthesis of hierarchical TS- 1 zeolites developed by Serrano et al. ${ }^{[16]}$

The top-down preparation routes for hierarchically structured TS1 consist mostly of post-synthetic demetallation treatment in alkaline or fluoride media to generate mesopores by extraction of framework atoms. ${ }^{[17]}$ Often, the demetallation step is followed by a subsequent recrystallisation step to increase the crystallinity and titanium incorporation of the formed hierarchically structured zeolite. [18] All of these preparation techniques result in hierarchical TS-1 zeolites with additional mesopores. Preparation routes for hierarchical macroporous TS-1 zeolites are not well known in literature.

Regardless of the choice of synthesis method, thorough characterisation of the pore structures in novel zeolitic materials is essential to understand the effects of the chosen synthesis route on the resulting material, and therefore to promote rational catalyst design. [19] While standard methods such as $\mathrm{Hg}$ porosimetry, gas adsorption isotherms or BET offer some data on the pore network, this is limited to bulk or average measurements. Information about the morphology and pore structure of zeolitic materials down to the micropore range $(<2 \mathrm{~nm})$ can be obtained using transmission electron microscopy (TEM). Nanoscale tomographic imaging using electrons ${ }^{[20]}$ and more recently hard $X$-rays ${ }^{[21]}$ constitute powerful non-invasive options for highresolution three-dimensional (3D) imaging of porous features in catalyst materials. Tomography can measure and co-locate the sample pore network in 3D space and in a non-invasive manner, which is critical given the often delicate structure of hierarchical catalysts.

In the present work, we introduce a synthesis route for hierarchically structured TS-1 crystals with intracrystalline macropores by steam-assisted crystallisation (SAC) of mesoporous silica particles after impregnation with a titanium source and the structure-directing agent. The resulting hierarchical micro-macroporous TS-1 was characterised extensively with different techniques, including advanced 3D characterisation by electron tomography (ET) and ptychographic $\mathrm{X}$-ray computed tomography (PXCT). This allowed direct calculation of pore volume fractions, surface area and global composition on the single particle scale ( 1-2 $\mu \mathrm{m}$ diameter). Kinetic studies were carried out to investigate the formation mechanism of the macroporous crystals. Further, the titanium content in the samples was varied in a wide range during synthesis. In order to prove the catalytic activity of the titanium species present in the synthesised hierarchical TS-1 samples, the activity was tested in the epoxidation of 2-octene and compared to a conventional nano-sized TS-1 catalyst.

\section{Results and Discussion}

\section{Synthesis of hierarchically structured TS-1 with intracrystalline macropores}

For the synthesis of the macroporous TS-1 samples, mesoporous silica particles were used as sacrificial precursor particles and silica source. The synthesised mesoporous silica exhibited uniform, non-agglomerated particles of spherical morphology (Figure $1 \mathrm{~b}$ ). The particle size distribution was relatively narrow with an average diameter of $600 \mathrm{~nm}$.

The powder X-ray diffraction (XRD) pattern shows a broad (100) reflection at $2.4^{\circ} 2 \vartheta$ and a (110) reflection at $4.5^{\circ} 2 \vartheta^{.22]}$ The (200) and (210) reflections are not visible in the XRD pattern. This type of pattern was attributed to hexagonal ordered mesoporous silica with poorly ordered mesopore channels. ${ }^{[23]}$ The adsorption isotherm (see ESI) is of type IV characteristic for mesoporous materials. The BET surface area of $679 \mathrm{~m}^{2} \mathrm{~g}^{-1}$, as well as the total pore volume of $0.50 \mathrm{~cm}^{3} \mathrm{~g}^{-1}\left(\mathrm{p} / \mathrm{p}_{0}=0.99\right)$, are comparable to the data reported by Gao et al. ${ }^{[24]}$
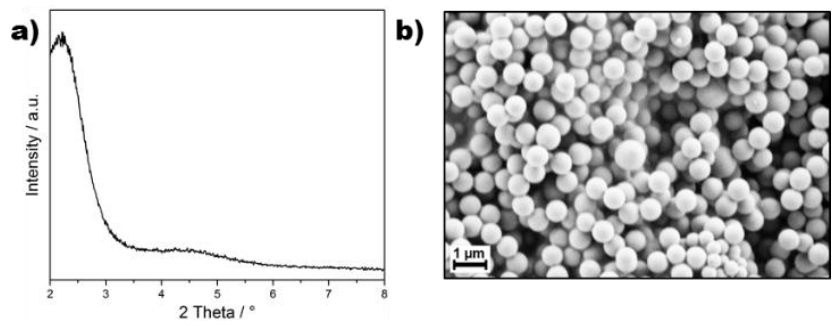

Figure 1. a) Powder XRD pattern and b) SEM micrograph of synthesised mesoporous, spherical silica particles (MSPs).

The synthesised MSPs were used as a precursor for the synthesis of macroporous TS- 1 zeolites by SAC. The addition of the titanium source was carried out by wetness impregnation of the calcined MSPs with the organic titanium source dissolved in isopropanol to give a molar ratio of $\mathrm{Si}$ to $\mathrm{Ti}$ of 80 . After drying, the Ti-MSPs were 
impregnated with an aqueous solution of the structure-directing agent (tetra propyl ammonium hydroxide, TPAOH). The two-step impregnation process was necessary due to the precipitation of the titanium source in aqueous media. The impregnated and dried MSPs were then converted into MFI zeolite by crystallisation under a steam atmosphere at elevated temperature and autogenous pressure.

The XRD pattern of the sample after SAC shown in Figure 2 reveals XRD lines typical for an MFI type zeolite with no indication of impurities. The $\mathrm{N}_{2}$-sorption isotherm shown in Figure 3 a) is of IUPAC type I, typical for microporous materials. The micropore volume of $0.174 \mathrm{~cm}^{3} \mathrm{~g}^{-1}$ is close to the theoretical micropore volume of an MFI zeolite and in good accordance to the XRD results. The crystals show a more rounded coffin shape known for MFI zeolites and external macropores in the range of 300 to 450 $\mathrm{nm}$. Those intracrystalline macropores are evenly distributed over the whole sample and present inside the crystals as evident in the TEM images in Figures $2 \mathrm{c}$ ) and d). The macroporosity appears to differ slightly from crystal to crystal. Figure $2 \mathrm{~d}$ ) shows a BFTEM image and the corresponding selected area electron diffraction (SAED) pattern in correct relative orientation, respectively. The diffraction pattern is consistent with the MFI crystal structure viewed along [010] in two superimposed orientation variants and thereby reveals that the particle is not an aggregation of many differently oriented crystals.
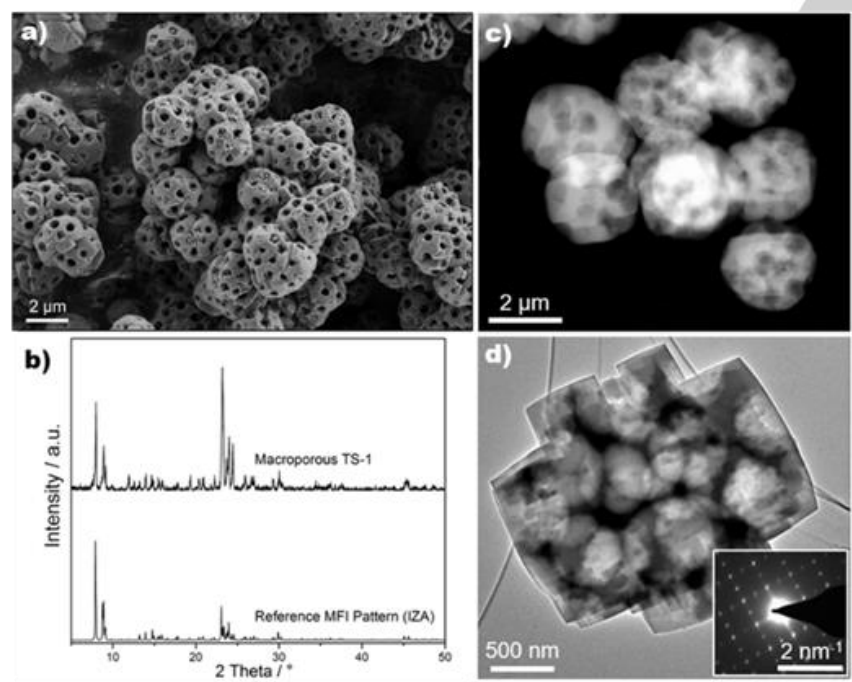

Figure 2. a) SEM image, b) powder XRD Pattern and c) HAADF STEM image of the calcined macroporous TS-1 sample. d) BF-TEM image of a single particle (macroporous TS-1) exhibiting strong crystal facets - the inset shows a SAED pattern with the same relative orientation of this particle consistent with the MFI crystal structure viewed along [010]MFI in two superimposed orientation variants.

Inductively-coupled plasma optical emission spectrometry (ICPOES) analysis shows a silicon to titanium ratio of 77 , close to the ratio in the impregnated MSPs before crystallisation. The diffuse reflectance UV-Vis spectrum of the calcined sample shown in Figure $3 \mathrm{~b}$ ) proves the incorporation of isolated tetrahedral titanium species $\left(205-210 \mathrm{~nm}\right.$, corresponding to $\mathrm{Ti}(\mathrm{OSi})_{4}$ species) and titanol species $\left(\mathrm{Ti}(\mathrm{OH})(\mathrm{OSi})_{2}\right.$ at $\left.228 \mathrm{~nm}\right)$ into the zeolite framework. Beside tetrahedral titanium species, the UVVis spectrum also shows a small amount of extra framework titanium species $\left(\mathrm{TiO}_{6}\right.$ isolated) as a broad peak from 240 to 260 $\mathrm{nm}$ as well as anatase like $\mathrm{TiO}_{2}$ species in the range of 325 to 340 $n m .{ }^{[25]}$
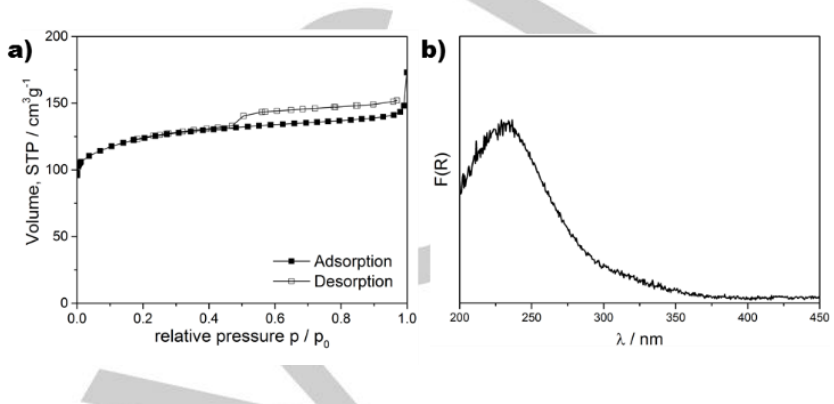

Figure 3. a) Nitrogen sorption isotherm and b) DR-UV-Vis spectrum of calcined macroporous TS-1.

In summary, the sample is of MFI topology with high crystallinity as evident by the XRD and $\mathrm{N}_{2}$-sorption data. Titanium is present in the sample and incorporated into the zeolite framework. The additional intracrystalline macropores are accessible from the bulk phase and present inside the crystal. These results prove the successful synthesis of hierarchically structured macroporous TS1. Furthermore, the SAED pattern is consistent with the MFI crystal structure in two superimposed orientation variants (viewed along [010]MFI) and therefore shows that the particle is not built by many differently oriented, inter-grown smaller crystals.

\section{Electron tomography and ptychographic X-ray computed tomography}

Following synthesis and physical characterisation, the TS-1 particles were investigated by 3D imaging, to identify and map interior porous features within the crystals. Figure 4 shows slices through the reconstructed ET dataset and a 3D volume rendering of one macroporous TS-1 crystal. The complete particle has a porosity of $33.6 \%$, determined using the maximum sphere inscription (MSI) algorithm applied to the segmented pore space (overall threshold) of the 3D reconstruction. The value is smaller than the $45 \%$ macroporosity reported for macroporous silicalite1. [26] The video of the electron tomography reconstruction provided in the supporting information shows that most of the pores are interconnected to each other with only some isolated macropores visible inside the crystal.

Interestingly, some interior macropores contain material which most probably is crystalline (partly faceted pieces with MFI crystal structure) see Figure 4 a) top right macropore. The presence of this material may indicate the growth of the zeolite into the macropores during synthesis. The fast dissolution of some of the MSPs could compromise the macropore template effect of the MSPs and result in crystal growth into the macropore. 

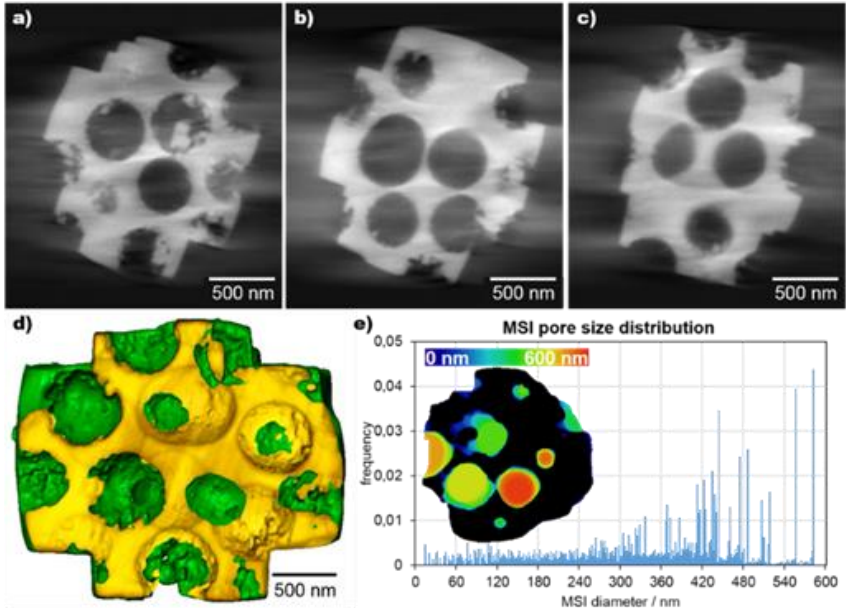

Figure 4. Electron tomography data of the macroporous TS-1 sample: a-c) Slices through the reconstructed volume (zeolite phase in light grey); d) 3D surface and volume rendering of the reconstructed volume with an open cut through the particle (yellow: surface facing towards zeolite; green: surface facing towards the pore and the empty surrounding space); e) volume-based pore size distribution (relative frequency of voxels with a certain assigned pore diameter with respect to the total number of pore voxels, i.e., total pore volume) derived from the MSI analysis - the inset shows an exemplary slice through the reconstruction (zeolite phase is black) with the colour-coded results of the MSI analysis.

Figure 4 e) shows the volume-based pore size distribution (PSD) derived from the MSI analysis. The peaks of bigger MSI diameters above $300 \mathrm{~nm}$ can be assigned to the individual macropores. The smaller pore diameters represent pore connections, pore necks towards the empty space surrounding the crystal and regions where the macropores contain smaller zeolite pieces.

The zeolitic walls visible in the slices through the ET reconstruction of the TS-1 crystal (Figure 4 a-c) are between 75 and $400 \mathrm{~nm}$ thick. In comparison to the macroporous silicalite-1 reported by Machoke et al., the synthesised macroporous TS-1 shows a higher wall thickness between the macropores. ${ }^{[26]}$

Another crystal of the same sample was also investigated using hard X-ray PXCT. PXCT is a phase contrast imaging technique which is sensitive to local electron density in the sample, and despite the use of hard X-rays can therefore be used to image low atomic number materials such as zeolites (e.g. $\mathrm{Si}, \mathrm{Al}, \mathrm{Ti}$ ), providing location and identification of structural components. Figure 5 shows the reconstructed TS- 1 particle observed by PXCT. The 3D spatial resolution of the volumes is approximately $47 \mathrm{~nm}$. The residual glue commonly resulting from focused ion beam (FIB) preparation can be easily detected and segmented out of the $3 \mathrm{D}$ volumes. To distinguish interior and exterior macroporosity, the pore features were labelled separately from the surrounding air and are indicated by the orange blobs in Figure 5. From slice-by-slice analysis of several cross-sections (Figure $5 \mathrm{~b}$ ) and transparency renderings (Figure $5 \mathrm{c}$ ), a variety of pore features can be observed within the particle. These include macropores breaching the particle surface, fully formed and partially formed macropores within the catalyst body. Notably, the PXCT data also shows presumably crystalline material in some of the internal macropores. The presence of surface-breaching pores and material in the macropores is important to note when planning the synthesis of hierarchical materials with specific structural elements and indicates how tomography may be used to tune particle size and porosity for the required purpose.

Volumetric data on the pore geometries was analysed and percentage macroporosity in the particle estimated at around $27 \%$ over the complete particle volume, again significantly lower than the $45 \%$ macroporosity observed for silicalite-1 ${ }^{[26]}$, but in general agreement with the ET results (33.6\%). The rather low measured macroporosity is probably due to partial zeolite growth into the macropores as noted previously, while the difference in values observed from ET can likely be attributed to natural variation among different particles. Furthermore, the spatial resolution of the measurement also has a strong effect of voxel counting used to determine percentage porosity, where it is expected that ET currently offers superior spatial resolution and therefore more accurate counting

(a)
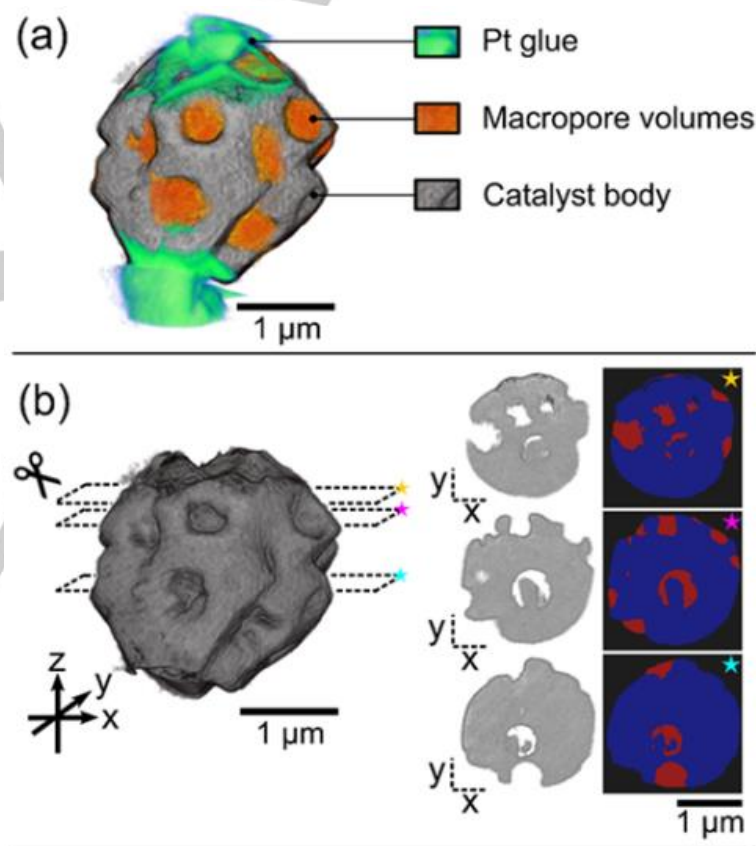

(c)
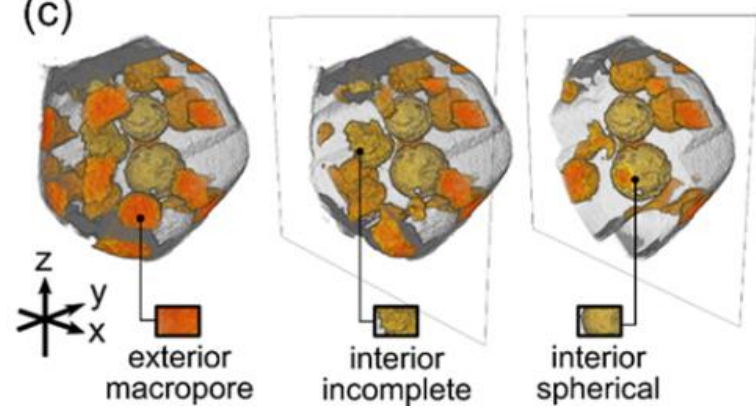

Figure 5. 3D renderings of a single TS-1 particle by PXCT showing: (a) segmentation and labelling of Pt glue (FIB contaminant), macropores and zeolite body; (b) orthographic projection of the slices indicated, with labelling of interior/exterior macropores (red); (c) transparent rendering showing variation in porous features present. 
of the pores present. At the same time, it was possible to simulate the effects of complete macropore formation by labelling the internal pores observed during PXCT as solid spheres, in which case observed macroporosity rose to around $45 \%$. It is again important to emphasise that while internal pore volumes can be measured by other techniques, only tomographic imaging (by electrons or X-rays) is capable of attributing the measured value to a physical 3D shape, and to reveal the presence of different types of pores, distance between pores, and distance from pores to surface of the particle. Especially the presence of crystalline material grown into the macropores would be hard to detect without the use of tomography.

The fundamental advantage of all tomography measurements for catalyst characterisation is the ability to not only obtain quantitative information on porosity (for example), but to localise these features in 3D space within the particle.

Ptychography in particular is a highly valuable emerging method from the synchrotron-based X-ray microscopy toolkit. By scanning a nanofocused beam across the sample and recording far-field diffraction patterns with an overlap at each measurement point, ptychographic image reconstruction can obtain unprecedented spatial resolutions which are not limited by the spot size of the incident beam. For example, PXCT has demonstrated spatial resolution as low as $15 \mathrm{~nm}$ for an integrated circuit sample, [27] and $23 \mathrm{~nm}$ for a nanoporous gold catalyst. ${ }^{[28]}$ Beyond superior spatial resolution, ptychography also allows derivation of local electron density in the probed sample, which can be calculated from the measured phase shift. ${ }^{[29]}$ Since electron density is generally unique to each chemical species, this can allow quantitative analysis of individual catalyst constituents from PXCT data. This is demonstrated in Figure 6, where a single slice from the tomogram is shown as a function of electron density (rather than phase shift data shown in Figure 5). As the electron density of any known species can be calculated from common tabulated mass density values, this allows the image histogram to be directly labelled with constituent materials. The images and histogram were offset so that voxels containing air have a phase shift of zero. In this case, the zeolite peak maxima $\left(0.75 \AA^{-3}\right)$ were an excellent match for the calculated values $\left(0.79 \AA^{-3}\right)$. The visible broadening of both the air and zeolite peaks follows a Gaussian distribution and may be attributed to partial volume effects resulting from features below the resolution limit, for example the zeolite micropores. In this case the resolution was insufficient to clearly distinguish macro- and micropores (which may be considered as air, i.e. normalised electron density of zero) from solid material. Likewise, the presence of minor Pt features $(>1 \AA$ $\left.{ }^{3}\right)$ was noted much lower than the calculated values $\left(5.1 \AA^{-3}\right)$, however these calculations were based on pure Pt, whereas FIBbased Pt deposition often use Pt-based compounds or mixtures.

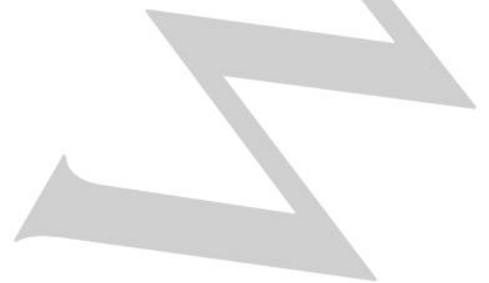

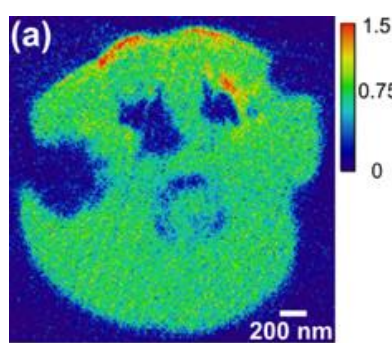

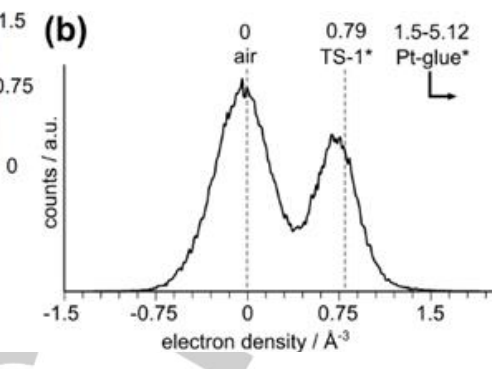

Figure 6. (a) Orthographic projection of a single slice of TS-1 obtained by PXCT colour bar indicates electron density showing zeolite (green) and minor Ptbased deposits (red); (b) histogram of the same slice, showing the measured electron density values of the individual catalyst constituents, calculated values indicated by grey lines. *For the purposes of estimating electron density, TS-1 was assumed to consist of pure $\mathrm{SiO}_{2}$, and Pt-glue of pure Pt.

Nevertheless, the ability to routinely present element/materialspecific electron density maps in both 2D images and 3D volumes offers excellent potential for catalyst characterisation using PXCT. While ET has typically been the established method for highresolution $3 \mathrm{D}$ imaging, the relatively high attenuation of the electrons limits the possible sample thickness and chemical composition (especially regarding heavier elements) that can be investigated by TEM and ET. The particle size, composition (lighter elements) and porosity (high porosity) of the investigated particles demonstrate that ET is still feasible on such larger structures. However, we are about to reach the limits of this technique. Although operating at significantly lower spatial resolution than electron microscopy, X-ray nanotomography using synchrotron radiation is rapidly coming into focus as a viable complementary method in catalysis research, allowing to investigate larger sample volumes and larger fields of view while providing inherent elemental specific information on the sample. ${ }^{[28,}$ 30]

\section{Kinetic investigation of the crystallisation mechanism}

For a better understanding of the crystallisation process, in particular with respect to the macropore formation mechanism, the kinetics of the crystallisation were studied by conducting experiments with different SAC times from 6 to 24 hours and a $\mathrm{Si} / \mathrm{Ti}$ ratio of the impregnated MSPs of 80 .

The sample after 6 hours at $150{ }^{\circ} \mathrm{C}$ was still amorphous without any XRD indication of a crystalline phase (see Figure 7 a). The first typical reflections for an MFI zeolite appear in the XRD pattern after 10 hours of SAC treatment. The crystallinity of the sample gradually increased over the synthesis time from 6 hours to 12 hours while the sample after 12 hours showed a complete crystallisation. 

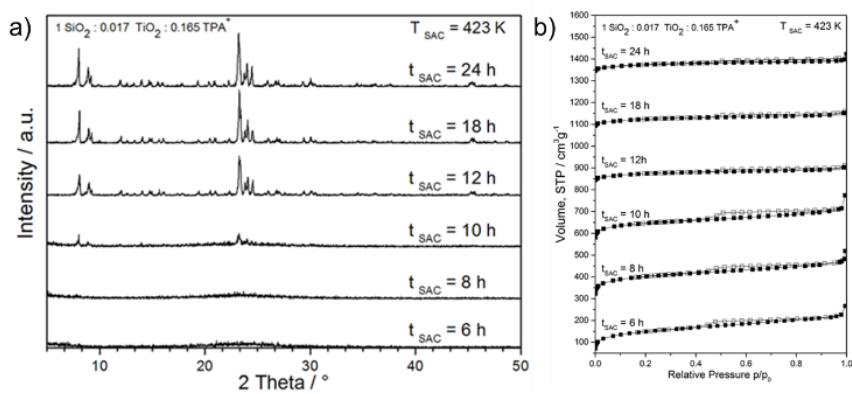

Figure 7. a) Powder XRD pattern and of samples after steam-assisted crystallisation for different times and b) Nitrogen sorption isotherms of samples after steam-assisted crystallisation for different times, off-set of $200 \mathrm{~cm}^{3} \mathrm{~g}^{-1}$.

The $\mathrm{N}_{2}$-sorption isotherms (Figure $7 \mathrm{~b}$ ) of the calcined samples show a transition from a type IV isotherm, characteristic for a mesoporous material to a type 1 isotherm characteristic for a microporous material with increasing synthesis time. During the synthesis, a mixture of type 1 and type IV isotherm indicates the presence of both micropores and mesopores in the samples.

The micropore volume apparently increased with crystallisation time due to the formation of MFI phase, while the total pore volume decreased with SAC time due to the dissolution of the mesoporous precursor particles (see Figure 8).

The crystallinity determined by integration of MFI typical XRD lines revealed the same trend, with drastic increase in crystallinity observed after 8 hours, followed by a plateau after 18 hours SAC, which indicates completion of the crystallisation process. By combining these results with the information in the scanning electron microscopy (SEM) images in Figure 9, it is possible to describe the crystallisation mechanism.
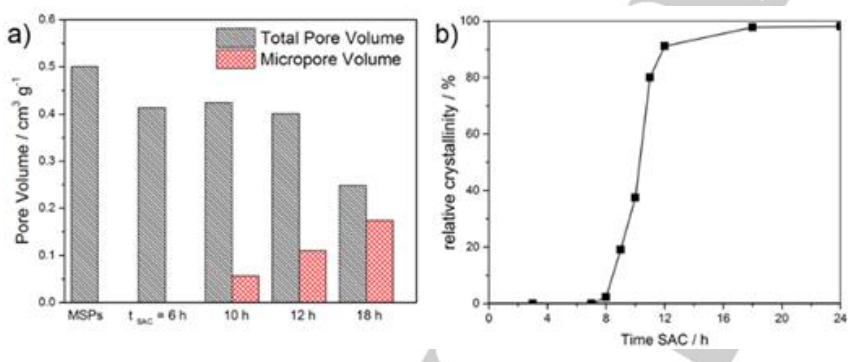

Figure 8. a) Total pore volume and micropore volume (alfa-S plot) of calcined samples and b) XRD crystallinity after steam-assisted crystallisation for different times.

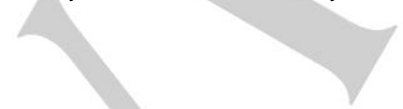

At the early stages of the synthesis, the MSPs were still intact, but it is likely that the dissolution of the MSPs already started. With further increase in SAC time, the dissolved silica reached supersaturation and nucleation of the zeolite started on the surface of the MSPs or in the intra-particle voids of the MSPs due to the presence of the structure directing agent (TPAOH) and the space necessary for the crystal formation. This is in good accordance with observations made for the synthesis of macroporous silicalite-1. ${ }^{[31]}$ The first crystals were visible in SEM after 8 hours SAC in between the MSPs, and the surface of the MSPs appeared rougher because of the enlargement of the mesopores due to dissolution (Figure $9 \mathrm{~b}$ ).

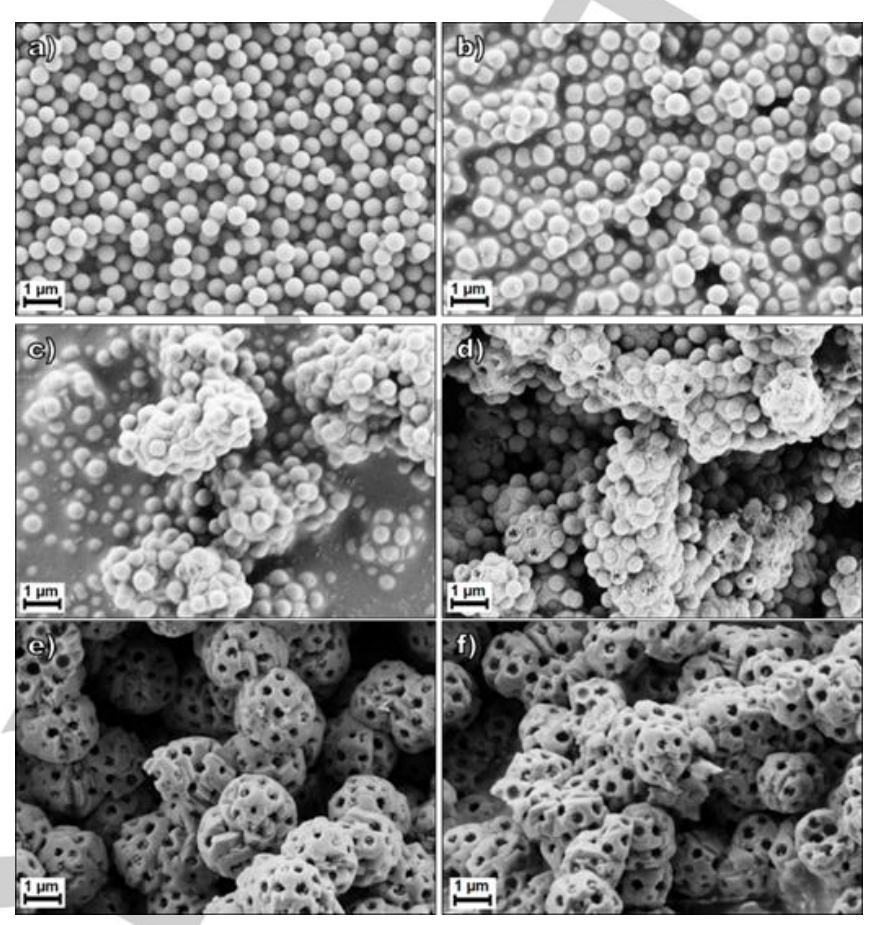

Figure 9. SEM micrographs of samples after steam-assisted crystallisation for different times; a) $6 \mathrm{~h}$, b) $8 \mathrm{~h}$, c) $10 \mathrm{~h}$, d) $12 \mathrm{~h}$, e) $18 \mathrm{~h}$ and f) $24 \mathrm{~h}$.

With further increase in the SAC time, the crystals grew and embedded more MSPs while the MSPs dissolution proceeded further resulting in larger pores visible in SEM (Figure $9 \mathrm{c}$ ) and evident in the nitrogen sorption data. The dissolution of the MSPs proceeds from the larger "inner" pore surface area rather than the small external surface area of the spherical particles, as indicated by the minimal shrinking of the MSPs and simultaneous increase in pore size of the MSPs. Therefore, the particles preserved their shape and size over the crystallisation process while becoming more porous. Consequently, the MSPs acted as a macropore template, preventing the growth of the zeolite at their location. Close to the end of the SAC process, the MSPs collapsed and dissolved completely resulting in macropores slightly smaller than the diameter of the precursor MSPs.

With prolonged synthesis time of up to 24 hours, no further changes to the material were evident (Figure $9 \mathrm{f}$ ). It should be noted that the MSPs visible in the SEM image of the sample after 12 hours (Figure $9 \mathrm{~d}$ ) show different states of dissolution. Some MSPs are dissolved almost completely while others are still intact. This inhomogeneity in dissolution rate of the MSPs can explain the crystal growth into some of the macropores evident in the ET and PXCT data. MSPs that are dissolved early cannot prevent the zeolite growth anymore, and the zeolites start to grow into the macropores. 


\section{Variation of the titanium content}

To study the influence of the titanium content on the synthesis of macroporous TS- 1 catalysts, the silicon to titanium ratio was adjusted during the impregnation to $100,80,60,40$ and 30 and the samples were crystallised under analogous conditions ( $423 \mathrm{~K}$, $24 \mathrm{~h})$.

Regardless of the titanium content, all synthesised samples were of MFI topology and exhibited high crystallinity as evident in the powder XRD pattern (see Figure $10 \mathrm{f}$ ).

The $\mathrm{N}_{2}$-sorption isotherms shown in Figure 11 are of type 1 characteristic for microporous materials. The BET areas range from 468 to $509 \mathrm{~m}^{2} \mathrm{~g}^{-1}$, and the micropore volumes are around $0.17 \mathrm{~cm}^{3} \mathrm{~g}^{-1}$, confirming the high crystallinity of the MFI zeolites for all studied titanium contents. The $\mathrm{Si} / \mathrm{Ti}$ molar ratios of the synthesised samples differed from the $\mathrm{Si} / \mathrm{Ti}$ ratios adjusted during the impregnation step (see Table 1). The samples with lower Ti content $(\mathrm{Si} / \mathrm{Ti} 100,80,60)$ showed values relatively close to the $\mathrm{Si} / \mathrm{Ti}$ ratio in the impregnated MSPs. With increasing amount of impregnated titanium-source, the difference between the $\mathrm{Si} / \mathrm{Ti}$ ratio in the dry gel and in the synthesised samples became more pronounced. The sample with the highest titanium content showed a Si/Ti ratio of 30 in the dry gel and 44 in the zeolite. This large difference could be attributed to the proposed maximal titanium content of about 2.2 titanium atoms per MFI unit cell by Millini et al. ${ }^{[32]}$ While the synthesis of TS-1 with higher titanium content has been reported in the literature, ${ }^{[33]}$ under the synthesis conditions used in the present work the maximum of 2.2 Ti per unit cell appears to be correct.

The SEM images shown in Figure 10 reveal intracrystalline macropores in all samples regardless of the titanium content. The crystal dimensions were similar for the low titanium containing samples and decreased when more titanium was added. SEM micrographs of the sample Macro TS-1 30 show nanoparticles visible on the crystal surface and in the bulk of the sample. The particles might be anatase $\mathrm{TiO}_{2}$ species, which would be in good accordance with the presence of a larger amount of anatase $\mathrm{TiO}_{2}$ evident in the DR-UV-Vis spectrum of the sample. However, the amount of anatase appears to be relatively low considering the absence of characteristic reflections in the XRD pattern.

The macropores visible on the crystal surface increased slightly in diameter with increasing titanium amount added in the impregnation step (average macropore diameter estimated by measuring 50 pores in SEM: Macro TS-1 $100=158 \mathrm{~nm}$, Macro TS-1 $80=194 \mathrm{~nm}$, Macro TS- $160=236 \mathrm{~nm}$, Macro TS-1 $40=$ $319 \mathrm{~nm}$, Macro TS-1 $30340 \mathrm{~nm}$ ). This might be an effect of the titanium precursor in the mesopores hindering TPAOH from entering the pores during the second impregnation step, resulting in a more pronounced egg-shell profile of the TPA ${ }^{+}$in the MSPs. However, in our previous work, we could show that the macropore diameter measured by TEM and SEM differ, due to the noncylindrical macropores. ${ }^{[34]}$

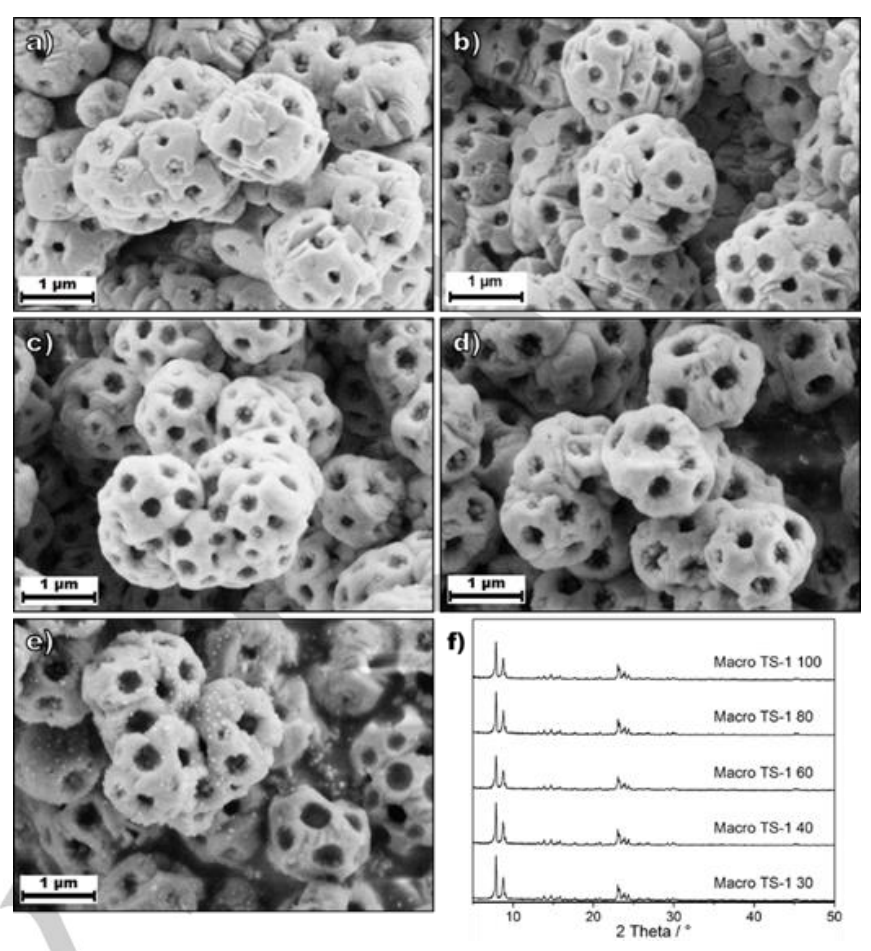

Figure 10. SEM Images of macroporous TS-1 samples with different titanium content; a) Macro TS-1 100 b) Macro TS-1 80, c) Macro TS-1 60, d) Macro TS140, e) Macro TS-1 30 and f) XRD pattern of the same samples.

While the crystallinity and the textural properties of the samples do not vary significantly, DR-UV-VIS spectra reveal differences in the characteristic peaks for the titanium species (see Figure $11 \mathrm{~b}$ ). The intensities of the peaks attributed to framework titanium species $\left(\mathrm{Ti}(\mathrm{OSi})_{4}\right.$ and $\mathrm{Ti}(\mathrm{OH})(\mathrm{OSi})_{2}$ at about 205 and $230 \mathrm{~nm}$, respectively) were found to increase with increasing titanium content. This is not the case of the sample with $\mathrm{Si} / \mathrm{Ti}$ set to 60 which shows a lower intensity than expected. The samples with high titanium content ( $\mathrm{Si} / \mathrm{Ti}$ set to 30 and 40 ) show comparable peak intensity. This is in good accordance with the similar titanium content in the samples evident in ICP-OES data.
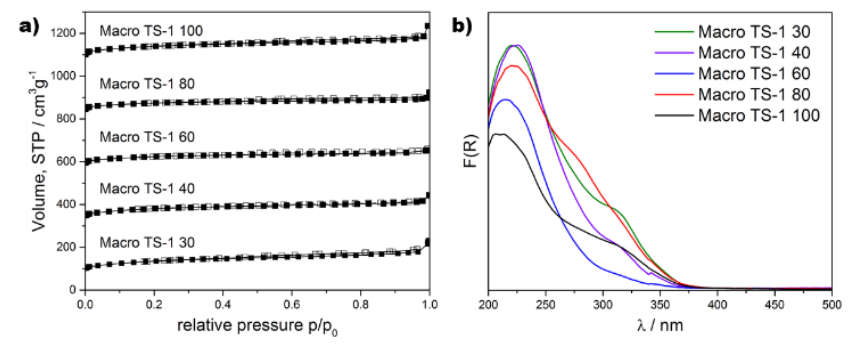

Figure 11. a) Nitrogen sorption isotherms (offset $200 \mathrm{~cm}^{3} \mathrm{~g}^{-1}$ ) and b) DR-UVVis spectra of calcined macroporous TS-1 samples with different titanium content. 
All UV-vis spectra show peaks attributed to extra-framework titanium species at about $240 \mathrm{~nm}\left(\mathrm{TiO}_{6}\right)$ and $330 \mathrm{~nm}$ (anatase $\mathrm{TiO}_{2}$ ). Inhomogeneity in the distribution of the titanium source could cause the formation of extra framework titanium species. High local titanium concentrations may exceed the maximum titanium amount in TS-1 (2.2 Ti/unit cell). Thus, the titanium surplus could form extra framework species. A distinct peak at $325 \mathrm{~nm}$ attributed to anatase like $\mathrm{TiO}_{2}$ is visible in the spectra of the sample with the highest impregnated titanium content (Macro TS-1 30). The presence of this larger amount of anatase could explain the nanoparticles visible in the SEM image (see Figure 10 e).

Table 1. ICP-OES and nitrogen sorption data of the synthesised TS-1 samples and the reference TS-1.

\begin{tabular}{lccccc}
\hline Sample & $\mathrm{Si}^{-\mathrm{Ti}_{\text {set }}{ }^{[a]}}$ & $\mathrm{Si} / \mathrm{Ti}{ }^{[\mathrm{b}]}$ & $\mathrm{Ti} / \mathrm{u} . \mathrm{C}^{[\mathrm{c}]}$ & $\mathrm{S}_{\mathrm{BET}}{ }^{[\mathrm{d}]}$ & $\mathrm{V}_{\text {micro }}{ }^{[\mathrm{e}]}$ \\
& - & - & - & $\mathrm{m}^{2} \mathrm{~g}^{-1}$ & $\mathrm{~cm}^{3} \mathrm{~g}^{-1}$ \\
\hline Conventional TS-1 & - & 48.0 & 1.99 & 498 & 0.173 \\
Macro TS-1-100 & 100 & 95.4 & 1.00 & 479 & 0.172 \\
Macro TS-1-80 & 80 & 77.7 & 1.24 & 468 & 0.174 \\
Macro TS-1-60 & 60 & 64.9 & 1.49 & 509 & 0.179 \\
Macro TS-1-40 & 40 & 48.1 & 1.97 & 488 & 0.176 \\
Macro TS-1-30 & 30 & 44.1 & 2.18 & 472 & 0.175 \\
\hline
\end{tabular}

[a] $\mathrm{Si} / \mathrm{Ti}$ ratio in the synthesis mixture, [b] $\mathrm{Si} / \mathrm{Al}$ ratio in the zeolite measured by ICP/OES, [c] Ti atoms per MFI unit cell calculated using the ICP data, [d] BET specific surface area determined by nitrogen sorption, [e] micropore volume determined by alfa-S-method.

\section{Catalytic performance in octene epoxidation}

The epoxidation of linear alkenes was studied to determine the activity of the hierarchically structured TS- 1 zeolites compared to a conventional TS- 1 catalyst. The conventional TS- 1 consists of crystals with nearly cubic morphology and dimensions of about 150 to $200 \mathrm{~nm}$. Detailed characterisation data of the conventional TS-1 can be found in the electronic supporting information. Both catalysts, conventional and hierarchical TS-1, showed comparable $\mathrm{Si} / \mathrm{Ti}$ ratios of around 48 . The observed 2-octene conversion increased over the reaction time for both catalysts. However, the macroporous catalyst showed uniformly higher activity at all temperatures tested than the conventional TS-1 catalyst (Figure $12 \mathrm{a}$ ). After 180 minutes, the conversion reached 55 and $73 \%$ for the conventional and macroporous TS- 1 samples respectively. A possible explanation for the observed difference in conversion is the varying degree of titanium incorporation into the framework of the TS-1 catalysts. The UV-Vis analysis (Figure ESI 4) indicated that a higher fraction of framework titanium species was present in the macroporous sample than in the conventional TS- 1 sample. Consequently the macroporous TS- 1 would exhibit a larger number of active sites than the conventional TS-1. Beside the number of active sites also the distribution of the titanium in the zeolites could be different. In future, EDX analysis of cross sections could help to determine possible differences in titanium distribution in the hierarchical and conventional TS-1 zeolite crystals.

The selectivity to the epoxide was nearly equal for both catalysts and followed the same trend. The relatively low selectivity of 42 to $45 \%$, shown in Figure 12 b), is in good accordance with similar measurements of alkene epoxidation over TS-1 reported in the literature. ${ }^{[35]}$ Beside the epoxide, only a very small amount of the diol was detectable in the reaction mixture after the reaction. Products adsorbed on the catalysts could cause the low selectivity. Another explanation could be the formation of products not detectable by the used analysis procedure, possibly larger molecules like glycol ethers.
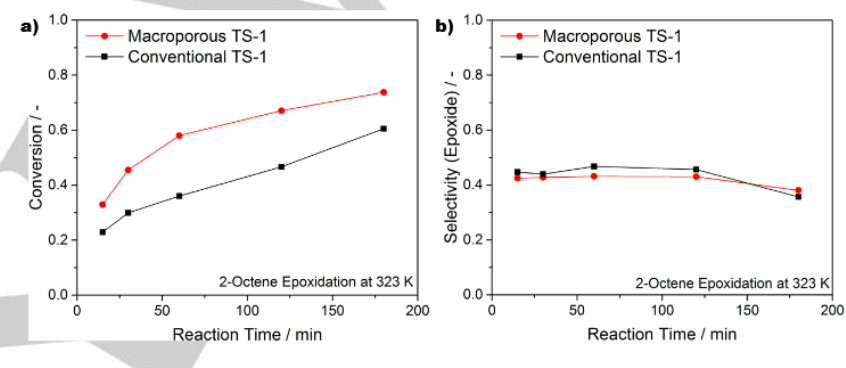

Figure 12. a) 2-Octene Conversion and b) Selectivity to the epoxide ove reaction time for macroporous and conventional TS-1 samples $\left(T_{\text {Reaction }}=323\right.$ $\mathrm{K})$.

Figure 13 shows the conversion and the selectivity to octene oxide of macroporous TS- 1 catalysts with different titanium content. With increasing titanium content the conversion of 2octene increased, with the exception of TS-1-30, indicating the presence of a higher number of active sites.
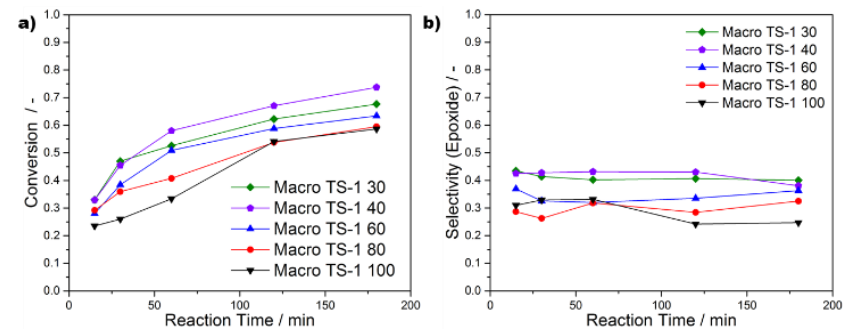

Figure 13. a) 2-Octene conversion and b) Selectivity to the epoxide over reaction time for macroporous TS-1 catalysts with different titanium content $\left(\mathrm{T}_{\text {Reaction }}=323 \mathrm{~K}\right)$.

The selectivity to octene oxide was relatively stable over the reaction time for all catalysts. With increasing titanium content in the catalysts, the selectivity to the epoxide increased from 30 up to $45 \%$. This might be an effect of the higher number of active 
sites for the epoxidation while other reactions like oligomerisation occur at the same reaction rate not influenced by the number of active sites present in the catalyst.

The presented catalytic studies were likely carried out under reaction limited conditions. Thus they do not allow insight in the different mass transport properties of the studied conventional and hierarchical TS-1 samples. To evaluate the effect of the macropores on the catalytic performance of TS-1 catalysts, additional future studies under diffusion limited reaction conditions are necessary.

\section{Conclusions}

Hierarchically structured TS- 1 single crystals with intra-crystalline macropores and a range of $\mathrm{Si} / \mathrm{Ti}$ ratios $(\mathrm{Si} / \mathrm{Ti}=95-44)$ were successfully synthesised by steam-assisted crystallisation of mesoporous silica particles. The titanium impregnated into the MSPs precursor particles was mostly incorporated into the zeolite framework during the synthesis. The resulting crystals show high macroporosity and highly crystalline MFI structure while DR-UVVis proves the incorporation of titanium into the zeolite framework. 3D tomographic imaging by ET and PXCT proved to be valuable tools for visualizing the hierarchical zeolites, i.e. the existence of a distributed macropore system within the crystal, which is connected to surface breaching macropores. The macroporosity of individual TS-1 crystals was estimated at 27 and $33 \%$ using PXCT and ET, respectively. The observed formation process of the obtained macroporous TS-1 crystals is in good accordance with the description of the formation of corresponding macroporous silicalite- 1 materials reported by Machoke et al. The results of the kinetic investigation emphasise the importance of MSP dissolution from their inner surface area for successful macropore formation. The zeolite crystal formation starts on the surface of the mesoporous particles, proceeds into the voids between the particles, and fills those completely. As a conclusion, one has to state that the silica particles fulfil more functions in this process, (i) act as sacrificial hard templates and (ii) provide the raw materials for the zeolite crystallisation. Thus, the resulting crystals represent the negative or the inverse geometry of the initial packing of the mesoporous silica particles, with macropores where the MSPs were previously located and zeolitic walls where the interparticle voids were located before crystallisation. We therefore propose the term "inverse crystallisation" as an appropriate name to describe the process. The macroporous TS1 was tested in 2-octene epoxidation and showed higher activity and comparable selectivity to a conventional nano-sized TS-1 catalyst. This proves the activity of the titanium species present in the synthesised macroporous TS- 1 samples. With higher titanium content, the conversion, as well as the selectivity to the epoxide, increased. The prepared catalysts have a highly crystalline character and an additional network of interconnected macropores in the crystals, accessible from the external surface. The combination should benefit the catalytic performance of the material by enhancing the mass transport due to the larger pores, while still preserving the shape selectivity and high activity of the crystalline TS-1. However studies of the titanium distribution in the macroporous TS-1 samples and investigations of their mass transport properties should be carried out to get a deeper understanding of their catalytic potential.

The reported synthesis of hierarchical zeolites, by inverse crystallisation of MSPs, allows the precise adjustment of the macropore diameter. ${ }^{[34]}$ This could be crucial for a rational catalyst design and might be beneficial for future systematic diffusion studies, aimed toward selective measurement of all contributions to overall mass transfer. ${ }^{[36]}$

Furthermore, the material consists of conventionally shaped and sized crystals, which might be beneficial for industrial applications due to easy handling and shaping during catalyst preparation and easy catalyst separation after the reaction. Therefore, the hierarchically structured, macroporous TS- 1 could be a potential novel catalytic material for industrially relevant reactions like the propylene oxide production by the HPPO process or the formation of cyclohexane oxime by ammoxidation of cyclohexanone.

\section{Experimental Section}

\section{Synthesis of Mesoporous silica particles}

Spherical mesoporous silica particles used as substrate for the preparation of macroporous zeolites were synthesised using the modified method of Gao et al. reported by Machoke and coworkers. ${ }^{[24]}$ In a typical synthesis, $2264.0 \mathrm{~g}$ ethanol (Merck) was mixed with $830.0 \mathrm{~g}$ deionised water in a $5 \mathrm{I}$ beaker with magnetic stirring. Thereafter $6.0 \mathrm{~g}$ hexadecyltrimethyl-ammonium bromide (CTAB, Sigma Aldrich) was added and dissolved, followed by addition of $130.5 \mathrm{~g} 25 \mathrm{wt}$. \% aqueous ammonia solution (Merck Emsure). The mixture was stirred for $1 \mathrm{~h}$ before $18.8 \mathrm{~g}$ tetraethyl orthosilicate (TEOS, Alfa Aesar) was added to give a molar composition of:

1 TEOS : $570 \mathrm{H}_{2} \mathrm{O}: 0.18 \mathrm{CTAB}: 543 \mathrm{EtOH}: 21 \mathrm{NH}_{3}$

The reaction was carried out under stirring $(250 \mathrm{rpm})$ at room temperature for $2 \mathrm{~h}$. After the synthesis, the solid product was recovered by centrifugation, washed 3 times with a water/ethanol mixture and dried overnight at $348 \mathrm{~K}$. To remove the surfactant the samples were calcined at $823 \mathrm{~K}$ for $6 \mathrm{~h}$ under $300 \mathrm{I} \mathrm{h}^{-1}$ air flow (heating ramp $=1.2 \mathrm{~K} \mathrm{~min}^{-1}$ ) in a muffle furnace.

\section{Synthesis of macroporous TS-1 catalysts}

The synthesis of macroporous zeolite crystals was carried out following a similar approach as reported in our previous work. ${ }^{[26]}$ In a typical synthesis, $1.0 \mathrm{~g}$ of calcined MSPs was added into 1.9 $\mathrm{g}$ of a $5.0 \mathrm{wt} . \%$ titanium butoxide solution in isopropanol, mixed with a spatula and then dried at $303 \mathrm{~K}$ for $3 \mathrm{~h}$. The dry powder was stored at room temperature overnight and then impregnated with $1.4 \mathrm{~g}$ of a 40.0 wt.-\% aqueous tetrapropylammonium hydroxide solution (TPAOH, Sigma Aldrich) and mixed with a spatula to give a molar composition of:

$1 \mathrm{SiO}_{2}: 0.02 \mathrm{TiO}_{2}: 0.16 \mathrm{TPAOH}$ 
Afterwards the impregnated MSPs were dried at $313 \mathrm{~K}$ for $1.5 \mathrm{~h}$ in a convection oven. The dry powder was crushed and distributed into cylindrical PTFE crucibles. The crucibles were placed on top of spacer tubes in $25 \mathrm{ml}$ PTFE-lined stainless steel autoclaves filled with $8.0 \mathrm{~g}$ deionised water. SAC was done at $423 \mathrm{~K}$ for 6 to $24 \mathrm{~h}$. After the crystallisation, the solid product was recovered by filtration, washed with a water/ethanol mixture and dried at $348 \mathrm{~K}$ for $12 \mathrm{~h}$.

All catalysts were calcined in a muffle furnace under $300 \mathrm{I} \mathrm{h}^{-1}$ air flow at $823 \mathrm{~K}$ to remove the structure directing agent (heating ramp $1.2 \mathrm{~K} \mathrm{~min}^{-1}$ ).

The reference TS- 1 catalyst was prepared by hydrothermal synthesis under static conditions following the procedure (method 1) reported by van der Pol and van Hooff with slight adjustment to the $\mathrm{Si} / \mathrm{Ti}$ ratio. ${ }^{[37]}$

\section{Catalytic tests in the epoxidation of 2-octene}

The catalytic tests were done following the procedure reported by Přech and co-workers. ${ }^{[35,38]}$ Before the catalytic tests, the catalysts were activated in a muffle furnace at $673 \mathrm{~K}$ under air flow for $0.5 \mathrm{~h}$ and then cooled down in a desiccator. The catalytic tests were carried out as batch experiments in $25 \mathrm{ml}$ two-neck round bottom flasks equipped with magnetic stirring and a reflux cooler.

$50 \mathrm{mg}$ of the catalyst was added to $6.0 \mathrm{ml}$ of acetonitrile, followed by addition of $150 \mathrm{mg}$ of the internal standard mesitylene (Fluka) and $300 \mathrm{mg}$ of 2-octene (Alfa Aesar, cis + trans). The reaction mixture was then heated to the desired temperature of $323 \mathrm{~K}$ before injection of hydrogen peroxide $(0.15 \mathrm{~g}, 30$ wt.- $\%$ aqueous solution, Sigma Aldrich) to initiate the reaction. During the reaction, samples were taken at regular intervals. The solid catalyst was separated using a syringe filter followed by centrifugation. Then the liquid samples were analysed using an Agilent 6850 GC equipped with a $20 \mathrm{~m}$ DB-5 column and a flame ionisation detector.

\section{Catalyst characterisation}

SEM micrographs were taken by using a Carl Zeiss ULTRA 55 microscope at a voltage of $3.0 \mathrm{kV}$ without any sample pretreatment. Powder XRD measurements were carried out using a Philips diffractometer equipped with a $\mathrm{Cu} \mathrm{Ka} X$-ray tube $(40 \mathrm{kV}$, $40 \mathrm{~mA}$ ). Nitrogen sorption isotherms were measured at $77 \mathrm{~K}$ using a Quantachrome Quadrasorb. Before the measurements, samples were degassed at $523 \mathrm{~K}$ for $12 \mathrm{~h}$ under vacuum. Ammonia temperature programmed desorption (TPD) experiments were conducted using a Porotec TPD/R/O 1100. Samples were pre-treated by degassing at $873 \mathrm{~K}$ under helium flow for $3 \mathrm{~h}$, before ammonia was adsorbed at $120^{\circ} \mathrm{C}$ for $30 \mathrm{~min}$. For the desorption measurements, the sample was heated to 873 $\mathrm{K}$ with a heating ramp of $10 \mathrm{~K} \mathrm{~min}^{-1}$. For elemental analysis, the samples were dissolved at $523 \mathrm{~K}$ for 30 minutes in a mixture of hydrofluoric acid and sulfuric acid. ICP-OES measurements were carried out using a Spectro Ciros CCD instrument. Diffuse reflectance UV-Vis spectroscopy measurements of calcined TS-1 samples were done using a Jasco V-650 spectrometer without any sample pre-treatment

PXCT was performed at the hard X-ray nanoprobe endstation of beamline P06 of the synchrotron radiation source PETRA III at DESY (Hamburg, Germany) ${ }^{[39]}$ using an incident photon energy of $9.00 \mathrm{keV}$. Single grains of $\sim 1-2 \mu \mathrm{m}$ diameter were placed on titanium tomography pins using FIB micromanipulation at DESY Nanolab (Hamburg, DE). The tomography pins were then placed on the beamline stage and aligned to the centre of rotation with respect to the beam. A Fresnel zone plate was used to focus a coherent X-ray beam to a spot size of $70 \mathrm{~nm}$. The sample was placed $1 \mathrm{~mm}$ behind the focal spot, resulting in a $2 \mu \mathrm{m}$ diameter beam profile (FWHM) on the sample. The beam was scanned across a field of view of $4 * 4 \mu \mathrm{m}^{2}$ with 10 horizontal and 10 vertical steps of $400 \mathrm{~nm}$ each, following which the sample was rotated. This process was repeated in $2^{\circ}$ steps over an arc of $180^{\circ}$, resulting in 90 unique projections. Diffraction patterns were recorded at each scanning position using an EIGER $\times 4 \mathrm{M}$ detector (DECTRIS, Switzerland) with $75 \mu \mathrm{m}$ pixel size positioned at a distance of $2310 \mathrm{~mm}$ downstream of the sample. The total acquisition time for one complete tomography dataset was around 7 hours.

Ptychographic reconstruction of each 2D projection was performed using the (e)PIE algorithm. ${ }^{[40]}$ Diffraction patterns were cropped to 512 by 512 pixels, leading to a pixel size of $8.3 \mathrm{~nm}$ in the reconstructed images. The reconstructed projections were first cropped to a field of view of $3.6^{\star} 3.6 \mu \mathrm{m}^{2}$ to remove artefacts created by areas at the edge of the field of view during ptychographic reconstruction. Afterwards, the projections were aligned relative to each other in horizontal and vertical direction before performing the tomographic reconstruction using the gridrec algorithm of the tomopy package. ${ }^{[41]}$ The resolution of the reconstructed volumes was estimated using Fourier Shell Correlation (FSC) to be $47 \mathrm{~nm}$ (half bit criterion). Following tomographic reconstruction, each 3D dataset was pre-processed using FIJI and visualised using Avizo 9.4 software (ThermoFisher Scientific, USA). Electron density maps were directly calculated from the phase shift values measured during ptychography, according to the method described by Diaz and co-workers. [29] High-angle annular dark-field (HAADF) STEM, bright-field TEM (BF-TEM), SAED and ET were performed using a FEI Titan Themis 300 operated at an acceleration voltage of $300 \mathrm{kV}$. ET was carried out in HAADF micro-probe STEM imaging mode, with a semi-convergence angle of the electron beam adapted and decreased to $0.7 \mathrm{mrad}$ for an increased depth of field with respect to the particle size of several micrometer. The particle powder was dispersed onto a Lacey carbon film on Copper TEM Grid (200 mesh), which was then fixed onto a Fischione Model 2020 Advanced Tomography Holder. A conventional ET tilt series was acquired in a tilt-angle range of $-76^{\circ}-+72^{\circ}$ with a tilt-angle increment of $1{ }^{\circ}$. Each projection was acquired with $1024^{\star} 1024$ pixels and a pixel size of $3.8 \mathrm{~nm}$. The 3D volume of the zeolite particle was reconstructed using the FEI Inspect 3D software using the Simultaneous Iterative Reconstruction Technique (SIRT) algorithm with 50 iterations and visualised using the software Avizo for FEl systems Materials Science 8.1.0. The 
resolution of the reconstructed volume was estimated to be $34 \mathrm{~nm}$ (half-bit criterion) by using the Fourier Shell Correlation (FSC) algorithm of the software Imagic FSC (Image Science Software $\mathrm{GmbH}$ ). Subtomograms from even and odd members of the tilt series, which were reconstructed using the weighted back projection (WBP) algorithm of the FEI Inspect 3D software, were used as input datasets for the FSC. An own implementation of the maximum sphere inscription (MSI) algorithm presented by Novák and co-workers ${ }^{[42]}$ was used to determine the pore size distribution of the reconstructed macroporous particle.

\section{Acknowledgements}

The authors gratefully acknowledge the support of Johnson Matthey and the Cluster of Excellence "Engineering of Advanced Materials" at the University of ErlangenNuremberg, which is funded by the Deutsche Forschungsgemeinschaft (DFG) within the framework of its Excellence Initiative. Furthermore, the DFG is acknowledged for financial support within the framework of the SPP 1570 (projects SP 648/4-3 and SCHW 478/23-3). Thomas Sheppard and Jan-Dierk Grunwaldt acknowledge the support by the BMBF-project "MicTomoCat" (05K16VK1). Jiři Čejka thanks the Czech Science Foundation for the Expro project (19-27551X). We acknowledge DESY (Hamburg, Germany), a member of the Helmholtz Association HGF, for the provision of experimental facilities. Parts of this research were carried out at beamline P06 of PETRA III. We further acknowledge Thomas Keller and Satishkumar Kulkarni for access to FIB at DESY NanoLab granted by BMBF under grant no. 5K13WC3.

Keywords: catalysis $\cdot$ crystallization $\cdot$ epoxidation • heterogeneous $\cdot$ nanostructures $\cdot$ zeolites

[1] M. Taramasso, G. Perego, B. Notari, Google Patents, 1983.

[2] J. Prech, Catalysis Reviews 2018, 60, 71-131.

[3] aP. Bassler, M. Weidenbach, H. Goebbel, Chem. Eng. Trans 2010, 21, 571-576; bP. Roffia, G. Leofanti, A. Cesana, M. Mantegazza, M. Padovan, G. Petrini, S. Tonti, P. Gervasutti, in Studies in Surface Science and Catalysis, Vol. 55 (Eds.: G. Centi, F. Trifiro), Elsevier, 1990, pp. 43-52.

[4] J. Jiang, J. Yu, A. Corma, Angewandte Chemie International Edition 2010, 49, 3120-3145.

[5] aL.-H. Chen, X.-Y. Li, J. C. Rooke, Y.-H. Zhang, X.-Y. Yang, Y. Tang, F.-S. Xiao, B.-L. Su, Journal of Materials Chemistry 2012, 22, 17381-17403; bD. Schneider, D. Mehlhorn, P. Zeigermann, J. Kärger, R. Valiullin, Chemical Society Reviews 2016, 45, 34393467

[6]

aW. Schwieger, A. G. Machoke, B. Reiprich, T. Weissenberger, T. Selvam, M. Hartmann, in Zeolites in Catalysis: Properties and Applications, The Royal Society of Chemistry, 2017, pp. 103-145; bM. V. Opanasenko, W. J. Roth, J. Cejka, Catalysis Science \& Technology 2016, 6, 2467-2484.

L. Tosheva, V. P. Valtchev, Chemistry of materials 2005, 17, 24942513.
[18] aN. Wilde, M. Pelz, S. G. Gebhardt, R. Glaser, Green Chemistry 2015, 17, 3378-3389; bB. Wang, M. Lin, X. Peng, B. Zhu, X. Shu, RSC Advances 2016, 6, 44963-44971.

[19] aS. Mitchell, N.-L. Michels, K. Kunze, J. Pérez-Ramírez, Nature Chemistry 2012, 4, 825; bJ. Zečević, K. P. de Jong, P. E. de

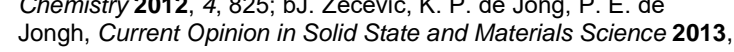
Jongh, Curre

[20] J. Zečević, C. J. Gommes, H. Friedrich, P. E. de Jongh, K. P. de Jong, Angewandte Chemie International Edition 2012, 51, 4213Jong,

[21] J. Ihli, R. R. Jacob, M. Holler, M. Guizar-Sicairos, A. Diaz, J. C. da Silva, D. Ferreira Sanchez, F. Krumeich, D. Grolimund, M. Taddei, W. C. Cheng, Y. Shu, A. Menzel, J. A. van Bokhoven, Nature Communications 2017, 8, 809.

[22] S. A. Bagshaw, E. Prouzet, T. J. Pinnavaia, Science 1995, 269, 1242-1244.

[23] G. Ozin, Journal of Materials Chemistry 1998, 8, 743-750.

[24] Q. Gao, Y. Xu, D. Wu, Y. Sun, X. Li, The Journal of Physical Chemistry C 2009, 113, 12753-12758.

[25] aP. Wu, T. Tatsumi, T. Komatsu, T. Yashima, The Journal of Physical Chemistry B 2001, 105, 2897-2905; bP. Ratnasamy, D. Srinivas, H. Knözinger, in Advances in Catalysis, Vol. 48, Academic Press, 2004, pp. 1-169; cF. Geobaldo, S. Bordiga, A. Zecchina, E. Giamello, G. Leofanti, G. Petrini, Catalysis Letters 1992, 16, 109-115; dT. Blasco, M. Camblor, A. Corma, J. PerezPariente, Journal of the American Chemical Society 1993, 115, 11806-11813.

[26] A. G. Machoke, A. M. Beltrán, A. Inayat, B. Winter, T. Weissenberger, N. Kruse, R. Güttel, E. Spiecker, W. Schwieger, Advanced Materials 2015, 27, 1066-1070.

[27] M. Holler, M. Guizar-Sicairos, E. H. R. Tsai, R. Dinapoli, E. Müller, O. Bunk, J. Raabe, G. Aeppli, Nature 2017, 543, 402.

[28] J. D. Grunwaldt, J. B. Wagner, R. E. Dunin - Borkowski, ChemCatChem 2013, 5, 62-80.

[29] A. Diaz, P. Trtik, M. Guizar-Sicairos, A. Menzel, P. Thibault, O. Bunk, Physical Review B 2012, 85, 020104.

[30] aY. Fam, T. L. Sheppard, A. Diaz, T. Scherer, M. Holler, W. Wang, D. Wang, P. Brenner, A. Wittstock, J. D. Grunwaldt,

ChemCatChem 2018, 10, 2858-2867; bA. M. Wise, J. N. Weker, S. Kalirai, M. Farmand, D. A. Shapiro, F. Meirer, B. M. Weckhuysen, ACS catalysis 2016, 6, 2178-2181. 
[31] Y. Zhang, L. Xu, J. Zhang, P. Li, Y. Yuan, H. Guo, X. Zhang, L. Xu, Catalysis Science \& Technology 2018, 8, 4526-4536.

[32] R. Millini, E. Previde Massara, G. Perego, G. Bellussi, Journal of Catalysis 1992, 137, 497-503.

[33] aA. Thangaraj, M. J. Eapen, S. Sivasanker, P. Ratnasamy, Zeolites 1992, 12, 943-950; bA. J. H. P. van der Pol, J. H. C. van Hooff, Applied Catalysis A: General 1992, 92, 93-111.

[34] T. Weissenberger, B. Reiprich, A. G. F. Machoke, K. Klühspies, J. Bauer, R. Dotzel, J. L. Casci, W. Schwieger, Catalysis Science \& Technology 2019, 9, 3259-3269.

[35] J. Přech, M. Kubư, J. Čejka, Catalysis Today 2014, 227, 80-86.

[36] aL. Gueudré, M. Milina, S. Mitchell, J. Pérez - Ramírez, Advanced Functional Materials 2014, 24, 209-219; bS. Mitchell, A. B. Pinar, J. Kenvin, P. Crivelli, J. Kärger, J. Pérez-Ramírez, Nature communications 2015, 6, 8633; cD. Schneider, D. Kondrashova, R. Valiullin, A. Bunde, J. Kaerger, Chemie Ingenieur Technik 2015 87, 1794-1809.

[37] A. Van der Pol, J. Van Hooff, Applied Catalysis A: General 1992 92, 93-111.

[38] J. Přech, D. Vitvarová, L. Lupínková, M. Kubů, J. Čejka, Microporous and Mesoporous Materials 2015, 212, 28-34.

[39] C. G. Schroer, M. Seyrich, M. Kahnt, S. Botta, R. Döhrmann, G. Falkenberg, J. Garrevoet, M. Lyubomirskiy, M. Scholz, A. Schropp, F. Wittwer, in SPIE Optical Engineering + Applications, Vol. 10389, SPIE, 2017, p. 10

[40] A. M. Maiden, J. M. Rodenburg, Ultramicroscopy 2009, 109, 12561262 .

[41] D. Gürsoy, F. De Carlo, X. Xiao, C. Jacobsen, Journal of Synchrotron Radiation 2014, 21, 1188-1193.

[42] V. Novák, F. Štěpánek, P. Kočí, M. Marek, M. Kubíček, Chemical Engineering Science 2010, 65, 2352-2360. 
Entry for the Table of Contents (Please choose one layout)

Layout 1:

\section{FULL PAPER}

Inverse crystallisation: Hierarchical titanium silicalite-1 zeolites with large intracrystalline macropores can be prepared by using mesoporous silica particles as sacrificial template. Interestingly, the zeolite growths in the interparticle voids around the steadily dissolving silica particles resulting in zeolite crystals, which represent the inverse of the initial packing of mesoporous particles.

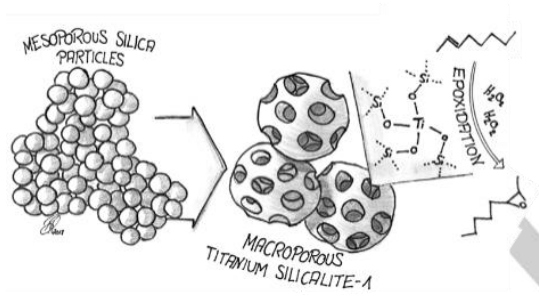

T. Weissenberger, R. Leonhardt, $B$. Apeleo Zubiri, M. Pitínová-Štekrová, T. L. Sheppard, B. Reiprich, J. Bauer. R. Dotzel, M. Kahnt, A. Schropp, C. G. Schroer, J. Grundwaldt, J. L. Casci, C. Čejka, E. Spiecker, W. Schwieger*

Page No. - Page No.

Synthesis and characterisation of hierarchically structured titanium silicalite-1 with large intracrystalline macropores 